\title{
ARTICLE
}

\section{Triggers of key calcium signals during erythrocyte invasion by Plasmodium falciparum}

Xiaohong Gao ${ }^{1}$, Karthigayan Gunalan¹, Sally Shu Lin Yap ${ }^{1} \&$ Peter R. Preiser ${ }^{1}$

Invasion of erythrocytes by Plasmodium falciparum merozoites is a complex multi-step process mediated by specific interactions between host receptors and parasite ligands. Reticulocytebinding protein homologues (RHs) and erythrocyte-binding-like (EBL) proteins are discharged from specialized organelles and used in early steps of invasion. Here we show that monoclonal antibodies against PfRH1 (an RH) block merozoite invasion by specifically inhibiting calcium signalling in the parasite, whereas invasion-inhibiting monoclonal antibodies targeting EBA175 (an EBL protein) have no effect on signalling. We further show that inhibition of this calcium signalling prevents EBA175 discharge and thereby formation of the junction between parasite and host cell. Our results indicate that PfRH1 has an initial sensing as well as signal transduction role that leads to the subsequent release of EBA175. They also provide new insights on how RH-host cell interactions lead to essential downstream signalling events in the parasite, suggesting new targets for malaria intervention.

\footnotetext{
${ }^{1}$ Division of Molecular Genetics \& Cell Biology, School of Biological Sciences, Nanyang Technological University, 60 Nanyang Drive, Singapore 637551 , Singapore. Correspondence and requests for materials should be addressed to P.R.P. (email: PRPreiser@ntu.edu.sg).
} 
lasmodium falciparum ( $P$. falciparum) is the most virulent malaria parasite and accounts for the vast majority of deaths (WHO, http://www.rbm.who.int, 2010). Erythrocyte invasion is a complex multi-step process that is mediated by specific interactions between host receptors and parasite ligands. Junction formation between the merozoite and erythrocyte is a crucial step that allows the parasite to irreversibly bind to the host followed by the activation of its motor machinery system that drives the parasite completely into the erythrocyte ${ }^{1}$. Previous studies suggest the involvement of apical secretory organelles termed rhoptries and micronemes ${ }^{2}$. In Plasmodium species, a number of key parasite ligands that mediate critical interactions with host receptors during invasion are localized in these apical organelles ${ }^{1}$. Two protein families are used in early steps of invasion in $P$. falciparum: reticulocyte-binding protein homologues (RHs) and erythrocyte-binding-like proteins (EBLs) ${ }^{3}$. RHs and EBLs have been shown to have a crucial role in host cell recognition and sensing as well as virulence ${ }^{4}$. Members of both protein families can be found in all Plasmodium species, further highlighting their importance in malaria parasite biology. In EBLs, a duffy-binding-like (DBL) domain mediates specific binding to different host cell receptors ${ }^{5}$ including glycophorins $\mathrm{A}, \mathrm{B}$ and $\mathrm{C}$ as well as the Duffy blood group antigen $^{3}$. In RHs, the precise erythrocyte-binding regions are still being delineated ${ }^{4,6-13}$; complement receptor 1 (CR1) and basigin have been identified to be the receptors for PfRH4 (ref. 14) and PfRH5 (ref. 15), respectively. Although RHs have been proposed to have an early role in host cell sensing ${ }^{16,17}$, the EBLs are thought to have a direct role in junction formation ${ }^{18}$. This suggests that $\mathrm{RH}$ sensing and subsequent interaction with a suitable host erythrocyte sends a signal to the merozoite that triggers the subsequent steps of invasion ${ }^{4}$.

Here we generate monoclonal antibodies (mAbs) against the erythrocyte-binding region RII-3 of PfRH1 (a member of the RH family in $P$. falciparum $^{7}$ ). We show that PfRH1 mAbs block merozoite invasion by specifically inhibiting calcium signalling in the parasite rather than by directly interfering with the erythrocyte binding of the parasite. We further show that the inhibition of this calcium signalling prevents EBA175 discharge and thereby prevents junction formation (a key step that enables the parasite to penetrate the erythrocyte using an actin-myosin motor $^{3}$ ). Our results demonstrate that PfRH1 and EBA175 have distinct functions, with PfRH1 having an initial sensing and signal transduction role that leads to subsequent EBA175 release from the microneme and to junction formation. These new insights might help to identify new targets for malaria intervention.

\section{Results}

Specific PfRH1 mAbs inhibit merozoite invasion. In this work, we use PfRH1 to study key intracellular signalling events that lead to successful erythrocyte invasion. We have previously shown that antibodies targeting an erythrocyte-binding region (RII-3) of PfRH1 (Fig. 1a) can inhibit merozoite invasion 7 . Here we generated four monoclonal antibodies (mAbs: C49, C41, C2 and $\mathrm{C50}$ ) against this region and validated their specificity using western blot and immunofluorescence assays. All antibodies recognize the expected 240 - and $140-\mathrm{kDa}$-processed form of PfRH1 in T994 parasite culture supernatant, merozoite and schizont extracts, whereas no equivalent bands were detected in the PfRH1 knockout line T994_RH1 (Supplementary Fig. S1a-c). Immunofluorescence assays showed that all $\mathrm{mAbs}$ give punctuate patterns that are partially localized with EBA175 (a known microneme protein) at the apical end of merozoites in T994, consistent with the expected location of PfRH1 (Supplementary

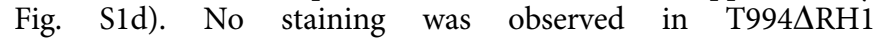
(Supplementary Fig. S1e), further confirming the specificity of the mAbs.

To establish whether the different mAbs were able to inhibit merozoite invasion, inhibition assays with increasing concentration of antibodies were carried out using T994 and T994DRH1 (Fig. 1b,c). We found that mAbs C2 and C50 have no significant effect on invasion, whereas both mAbs C49 and C41 inhibit invasion in a dose-dependent manner in T994. In contrast, none of the antibodies has any impact on T994 $\mathrm{RH} 1$, in line with their specificity to PfRH1. Similar results (Supplementary Fig. S2a,b) were obtained when using the parasite lines W2mef and W2mef/ $\mathrm{NM}$, which are known to differ in their requirement for PfRH1 in invasion $^{7,19}$, suggesting that both C49 and C41 are invasioninhibiting mAbs. To ensure that the invasion inhibitory effect is not because of the large size of the mAb (which could lead to a secondary blocking effect), we purified the Fab fragment of mAb C41 (Supplementary Fig. S2c, insert) and performed invasion
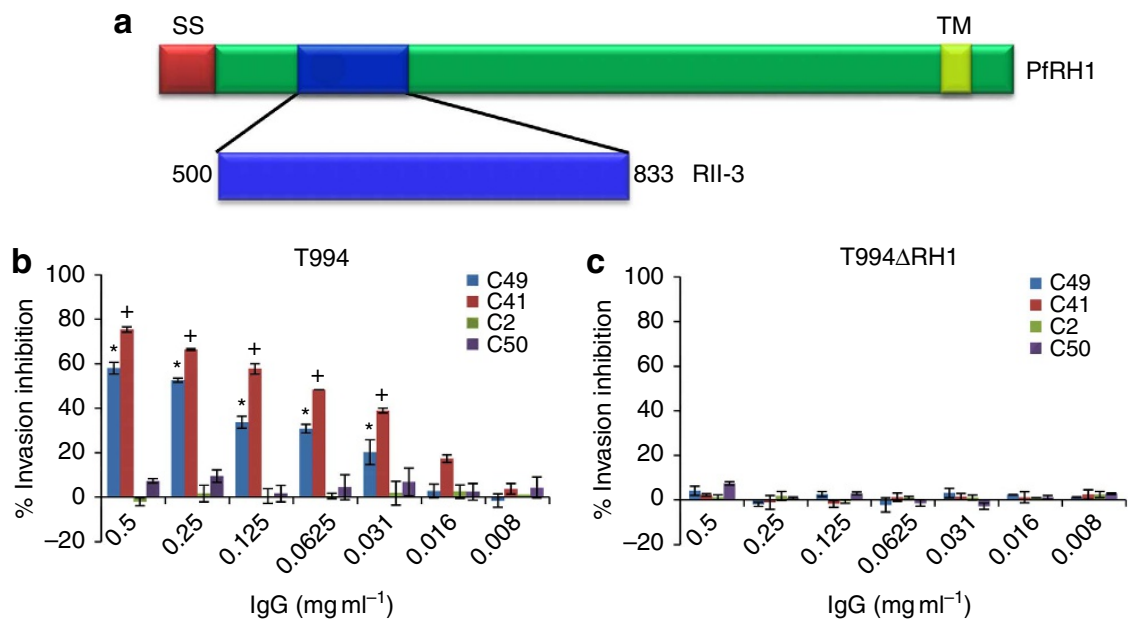

Figure 1 | Anti-PfRH1 mAbs significantly block parasite invasion. (a) Full-length PfRH1 including signal sequence (SS, red), erythrocyte-binding region RII-3 (blue, encompassing amino acids 500-833) and transmembrane domain (TM, yellow). (b,c) Invasion inhibition assays using anti-PfRH1 mAbs ( $\mathrm{lgG}$ ) in T994 (b) and T994 $\mathrm{RH} 1$ (c). Bar charts show mean \pm s.e.m.; $n=3$. ${ }^{\star} P \leq 0.0003$ by one-way ANOVA, indicating that C49 successfully blocked the invasion in $T 994$ compared with the invasion in the presence of $C 2$ and $C 50 .{ }^{+} P \leq 0.0003$ by one-way ANOVA, indicating that C41 significantly inhibited the invasion compared with $\mathrm{C} 2$ and C50. 
inhibition assays ${ }^{20,21}$. The purified C41 Fab fragment significantly blocks invasion in a dose-dependent manner in T994 parasites (Supplementary Fig. S2c), confirming that mAb C41 directly targets PfRH1.

Inhibitory mAbs block invasion before junction formation. Merozoite invasion inhibition by antibodies is often caused by antibodies directly interfering with the binding of the parasite to its receptor. We therefore assessed the ability of the different $\mathrm{mAbs}$ to inhibit PfRH1 binding to erythrocytes. Both non-inhibitory mAbs (C2 and C50) and inhibitory mAbs (C49 and C41), at concentrations $\left(0.3 \mathrm{mg} \mathrm{ml}^{-1}\right)$ shown to effectively inhibit merozoite invasion (Fig. 1b), have no impact on erythrocyte binding by PfRH1 (Fig. 2a,b), suggesting that invasion-blocking mAbs act independently of erythrocyte binding. To further determine the epitope specificity, four $\mathrm{N}$-terminal overlapping fragments (N1-N4) derived from RII-3 were expressed as recombinant proteins and screened using the mAbs (Supplementary Fig. S3). The size of each fragment was confirmed using anti-His-tag mAbs (Supplementary Fig. S3f). None of the PfRH1 mAbs recognizes $\mathrm{N} 1$, whereas $\mathrm{C} 2, \mathrm{C} 41$ and $\mathrm{C} 49$ recognize $\mathrm{N} 4$ only. $\mathrm{C} 50$ is able to detect $\mathrm{N} 2, \mathrm{~N} 3$ and $\mathrm{N} 4$ (Supplementary Fig. S3b-e), suggesting that C50 binds to a different epitope from that of $\mathrm{C} 2, \mathrm{C} 41$ or C49. On the basis of the western blot results, we further synthesized six overlapping peptides (Supplementary Fig. S3g). The inhibitory mAbs C41 and $\mathrm{C} 49$, as well as the non-inhibitory $\mathrm{mAb} C 2$, recognize the same 15-mer peptide, whereas another non-inhibitory mAb C50 recognizes a different peptide (Supplementary Fig. S3h). It is somewhat surprising that the non-inhibitory and inhibitory mAbs recognize the same peptide; however, as an epitope is $5-8$ amino-acid long, it is possible that multiple distinct epitopes lie within the same peptide. In fact, previous studies have shown that mAbs against epitopes that are very close to each other exhibit different effects on cellular or biological functions ${ }^{20-22}$.
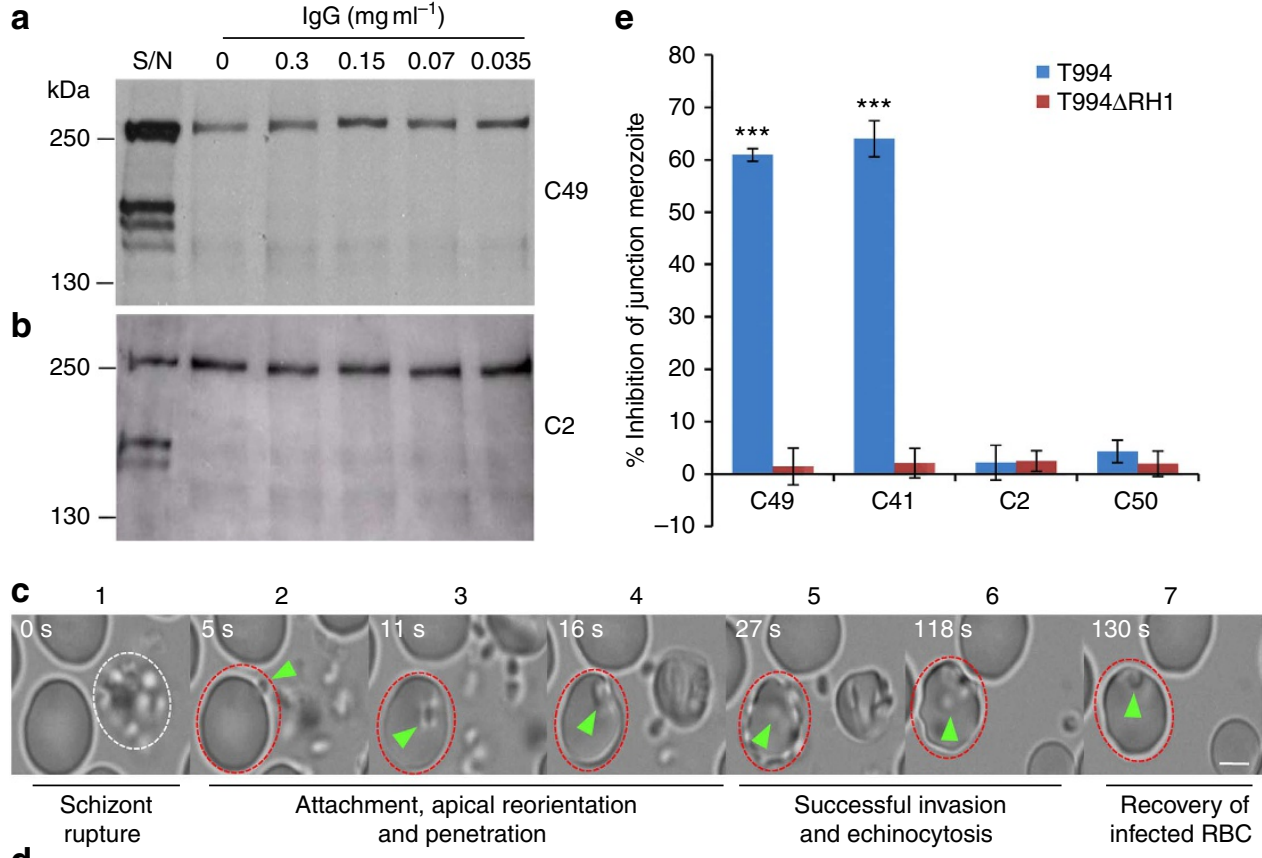

d

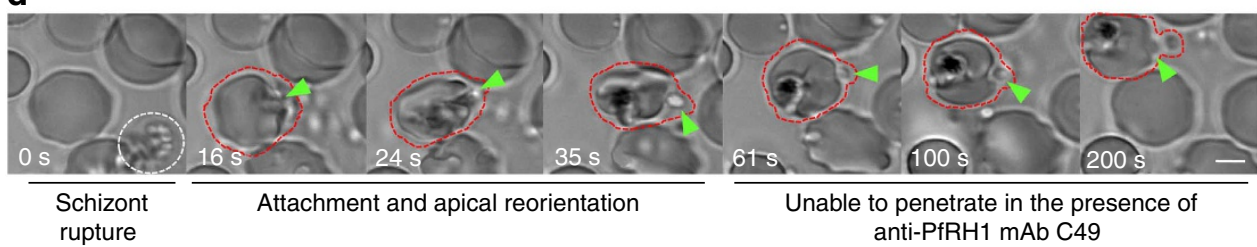

Figure 2 | Anti-PfRH1 mAbs inhibit invasion before junction formation. (a,b) Erythrocyte binding assays of T994 parasite culture supernatants using the C49 (a) and C2 (b) mAbs. Neither C49 nor C2 $\left(0.035-0.3 \mathrm{mg} \mathrm{ml}^{-1}\right)$ was able to inhibit the erythrocyte binding of PfRH1. T994 parasite culture supernatants $(\mathrm{S} / \mathrm{N})$ were used as a quality control. Molecular sizes are indicated on the left (in kDa). (c,d) Snapshots taken from time-lapse live movie microscopy of invasion of W2mef by merozoites in the absence (c) or presence (d) of C49 mAb. Time-elapsed post schizont rupture is indicated in each snapshot in seconds ( $\mathrm{sec}$, white). A white dotted circle indicates a rupturing schizont. Green arrows point to merozoites. A red dotted circle around an erythrocyte is added to help follow the infection. Scale bars $=5 \mu \mathrm{M}$. (c) In the absence of $\mathrm{mAb}$, the merozoite released from a mature schizont (white cycle) attaches, apically reorients and penetrates into an uninfected erythrocyte. Following successful invasion, deformation of infected erythrocytes occurs (echinocytosis) and by 2 min the erythrocytes recover back to its normal shape. (d) In the presence of C49, the merozoite released from a mature schizont attaches and apically reorients, but it is unable to penetrate the erythrocyte even after 3 min. Also see Supplementary Movies 1,2. (e) Anti-PfRH1 inhibitory mAbs significantly reduce merozoite junction formation. T994 or T994 $\mathrm{RH} 1$ schizonts were pretreated with Cyto D and allowed to rupture in the absence or presence of anti-PfRH1 mAbs $\left(0.2 \mathrm{mg} \mathrm{ml}^{-1}\right)$. Junction-arrested merozoites were counted microscopically. Bar chart indicates the counting of junction-arrested merozoites with mAbs C2, C41, C49 and C50 in T994 and T994 $\mathrm{RH} 1$ compared with the arrested parasites in the absence of mAbs. Inhibitory mAbs C49 and C41 significantly blocked merozoite junction formation in T994. The error bar indicates the s.e.m.; $n=3$. ${ }^{\star \star \star} P \leq 0.00014$ by one-way ANOVA indicates the significant differences between the effects of inhibitory mAbs (C49 and C41) and those of non-inhibitory mAbs (C2 and C50). 
Live video microscopy is a powerful tool to dissect merozoite invasion, and it has been effectively used to demonstrate the role of another merozoite invasion protein, PfAMA1, during merozoite re-orientation step in invasion ${ }^{23}$. Using time-lapse live video microscopy, we followed the process of merozoite invasion both in the presence and absence of invasion inhibitory antibodies (Fig. 2c,d, Supplementary Fig. S4a,b and Supplementary Movies 1-7). We found that, in the absence of antibodies, newly released merozoites rapidly attach, reorient and invade erythrocytes (Fig. 2c and Supplementary Movie 1). Immediately after invasion, the erythrocyte shrinks and echinocytosis occurs, with the infected erythrocyte recovering its shape within $2 \mathrm{~min}$. Interestingly, in the presence of the invasion inhibitory antibody C49 (Fig. 2d and Supplementary Movie 2,6) or C41 (Supplementary Fig. S4a and Supplementary Movie 3,7), merozoites are able to attach and reorient themselves but are unable to penetrate the erythrocyte even after $3 \mathrm{~min}$, despite extensive distortions of the erythrocyte being observed. In contrast, invasion similar to that seen in the absence of any antibody is observed in the presence of the non-inhibitory antibodies C2 (Supplementary Fig. S4b and Supplementary Movie 4) or C50 (Supplementary Movie 5). The inability of the merozoite to penetrate the erythrocyte despite reorientation and clear binding suggests that the inhibitory antibodies interfere with a subsequent step of the invasion process. Junction formation is known to be linked to the irreversible commitment of the merozoite to invasion, and recent studies have shown that PfRH1, $\mathrm{PfRH} 2 \mathrm{a} / 2 \mathrm{~b}$ and PfRH5 localize at the tight junction, suggesting a specific role for the PfRH proteins during this part of the invasion process $6,24-26$. Therefore, to assess whether the inhibitory mAbs prevented junction formation, merozoites were arrested at the tight junction by treatment with cytochalasin $\mathrm{D}(\text { Cyto } \mathrm{D})^{27}$. Experiments were carried out in the absence and presence of PfRH1 mAbs, and junction-arrested merozoites were counted. Similar to the invasion inhibition assay, inhibitory mAbs C49 and C41 significantly reduce junction-arrested merozoites as compared with C2 and C50 in both T994 and W2mef (Fig. 2e, Supplementary Table S1 and Supplementary Fig. S4c). In

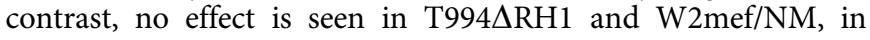
line with PfRH1 not having a role in these parasites. Taken together, these data clearly demonstrate that inhibitory mAbs inhibit the invasion before junction formation and suggest that PfRH1 could have a key signalling role ahead of junction formation.

PfRH1 inhibitory mAbs block $\mathrm{Ca}^{2+}$ signalling during invasion. Previous work has suggested that secretion of EBA175 from the micronemes onto the surface of the merozoite is a prerequisite for junction formation and is triggered by calcium $\left(\mathrm{Ca}^{2+}\right)$ release in the merozoite ${ }^{28}$. Here we carried out real-time measurement of $\mathrm{Ca}^{2}+$ signalling in the presence/absence of Cyto $\mathrm{D}$ during merozoite invasion using freshly isolated free merozoites from T994 parasite. We found that $\mathrm{Ca}^{2+}$ signal increases both in the presence and absence of Cyto D (Fig. 3a, Supplementary Data 1-6), demonstrating that the signal originates before or at junction formation. This allowed us to further elucidate whether the invasion inhibitory mAbs targeting PfRH1 directly had an impact on $\mathrm{Ca}^{2+}$ signalling. We observed that the cumulative intracellular $\mathrm{Ca}^{2}+$ signal during merozoite invasion is significantly reduced in the presence of the inhibitory mAbs C41, as compared with the non-inhibitory mAbs C2 or no antibody control (Fig. 3b, Supplementary Data 1-6). No antibody

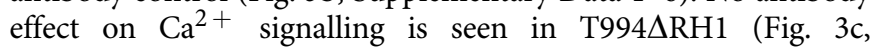
Supplementary Data 1-6). These results suggest that PfRH1 inhibitory mAbs inhibit invasion by blocking $\mathrm{Ca}^{2+}$ signalling.
Control experiments using the $\mathrm{Ca}^{2+}$ ionophore A23187 and the $\mathrm{Ca}^{2}+$ chelator BAPTA-AM showed that treatment of merozoites with A23187 leads to a significant increase in the $\mathrm{Ca}^{2}+$ signal over time, whereas pretreatment with BAPTA-AM significantly reduces $\mathrm{Ca}^{2+}$ signals (Supplementary Fig. S5, Supplementary Data 1-6 and Supplementary Table S2). Although the $\mathrm{Ca}^{2+}$ signal observed during invasion assays is only derived from successfully invading merozoites, all viable merozoites are expected to give $\mathrm{Ca}^{2+}$ signals after A23187 treatment, explaining the higher signal obtained (compare Fig. 3b with Supplementary Fig. S5). Importantly, functional C41 Fab fragments also block $\mathrm{Ca}^{2}+$ signalling in T994 parasite during invasion (Fig. 3d, Supplementary Data 1-6) as effectively as mAbs C41. This further confirms that the specific binding of inhibitory mAbs to PfRH1 is important for blocking $\mathrm{Ca}^{2+}$ signalling.

We performed similar experiments using flow cytometry to measure $\mathrm{Ca}^{2+}$ levels during invasion of fresh erythrocytes by free merozoites in the presence of Cyto D, as well as in the presence/ absence of inhibitory mAbs. Invading merozoites will be arrested by Cyto $\mathrm{D}$ after junction formation, whereas merozoites in the presence of inhibitory antibodies will either detach or remain attached to the RBC without forming a junction. On the basis of these criteria, there are two distinct populations: free merozoites and RBC-bound merozoites. Staining for DAPI $(+)$ events identifies all merozoites, whereas gating for size enables us to distinguish between free and RBC-bound merozoites (Fig. 3e,f). An inhibitory antibody $\mathrm{C} 41$ can reduce the number of invading merozoites to $56.6 \%$ (Fig. 3g-blue) and decrease the overall $\mathrm{Ca}^{2+}$ level to $32.42 \%$ (Fig. 3g-red) during invasion, when compared with the absence of inhibitory mAbs. This result is consistent with our previous conclusion, supporting the accessibility and reproducibility of real-time measurement of $\mathrm{Ca}^{2}+$ signalling by using a fluorescence plate reader. Taken together, these results strongly support the conclusion that PfRH1 has a direct or indirect role in the regulation of $\mathrm{Ca}^{2+}$ signalling during invasion. Hence, we propose that binding of the mAbs to PfRH1 directly inhibits a signal to trigger $\mathrm{Ca}^{2+}$ signal, or it has an indirect effect by preventing the recruitment of other proteins important for the initiation of the signal.

Merozoite viability. To ensure that the observed $\mathrm{Ca}^{2+}$ signal was indeed due to merozoite invasion, each merozoite preparation in parallel to $\mathrm{Ca}^{2+}$ signalling studies was assessed for their ability to invade fresh erythrocytes. T994 and T994 $\mathrm{RH} 1$ merozoites from each preparation successfully invade erythrocytes, and T994 invasion is successfully inhibited by $\mathrm{C} 41$ but not $\mathrm{C} 2$. In contrast, none of the antibodies has any impact on invasion in the case of T994 $\Delta$ RH1 (Supplementary Table S3). Importantly, there is a clear correlation $\left(R^{2}=0.836\right)$ between invasion and $\mathrm{Ca}^{2+}$ signal, with low invasion (due to antibody blocking) leading to low $\mathrm{Ca}^{2+}$ signal (Fig. 4a). In addition, we followed the kinetics of merozoite invasion using heparin treatment ${ }^{29}$ and observed that almost $75 \%$ of isolated merozoites invade erythrocytes in a linear manner $\left(R^{2}=0.991\right)$ over the first $10 \mathrm{~min}$ (Fig. $4 \mathrm{~b}$ ), which is in line with time-dependent $\mathrm{Ca}^{2+}$ data. Only a small proportion (25\%) of the total invading merozoites have not invaded within 10 min, confirming that the observed increase in $\mathrm{Ca}^{2}+$ signal was contributed by the majority proportion of invading merozoites over the first $10 \mathrm{~min}$. Taken together, these data clearly indicate that viable invasion-competent merozoites contribute to the signals.

An EBA175 inhibitory mAb has no effect on $\mathrm{Ca}^{2+}$ signalling. In line with published data, our results indicate that PfRH1 has a direct role in $\mathrm{Ca}^{2+}$ release, which then triggers microneme and 

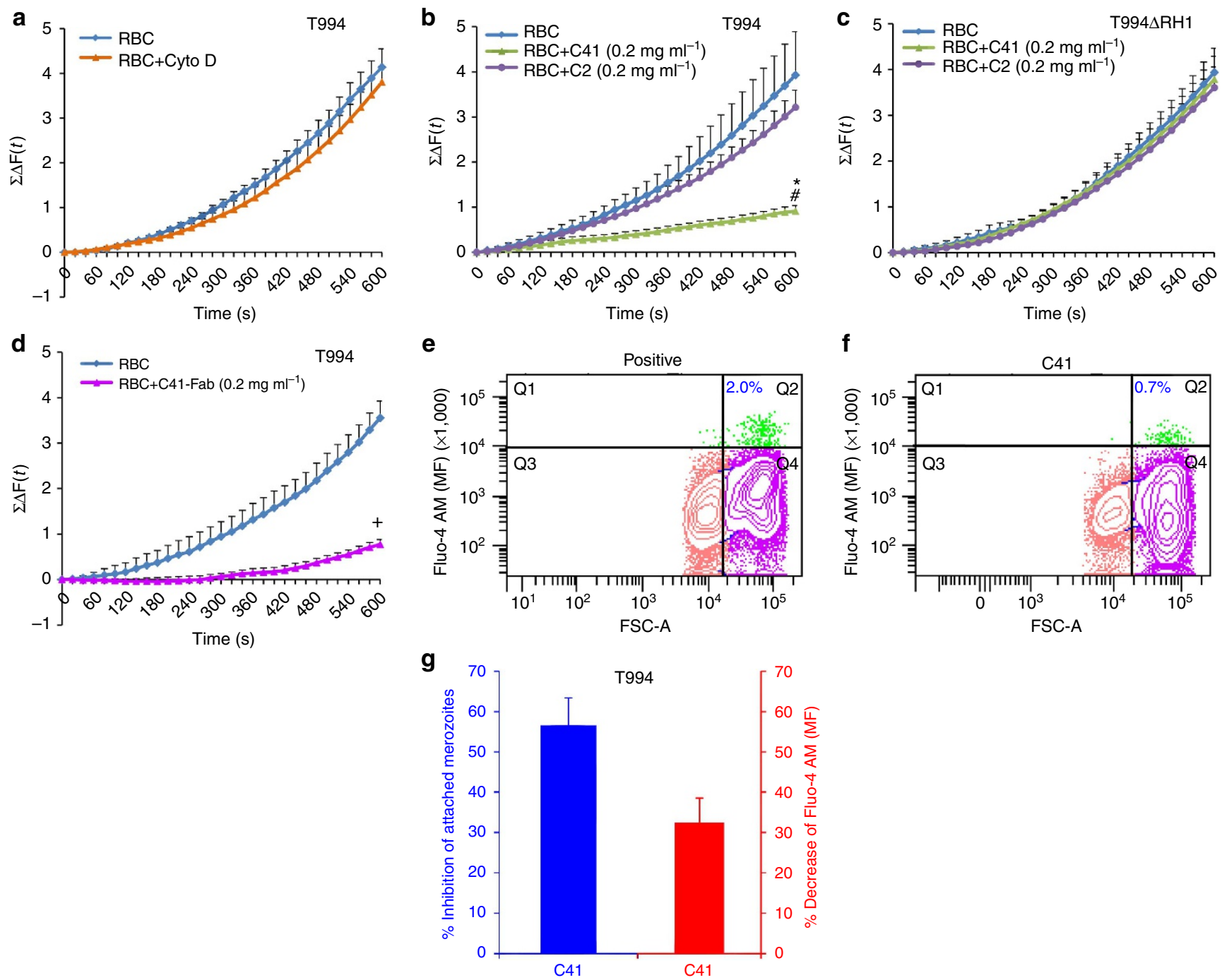

Figure 3 | Detection of cytosolic $\mathbf{C a}^{2}+$ levels during merozoite invasion. (a-d) Cytosolic $\mathrm{Ca}^{2+}$ levels were detected using a fluorescence plate reader. (a) Preloaded T994 merozoites with Fluo-4AM were incubated with erythrocytes in the absence (RBC) or presence of Cyto D (RBC+Cyto D).

$(\mathbf{b}, \mathbf{c})$ Preloaded T994 (b) or T994 $\mathrm{RH} 1$ (c) merozoites were incubated with erythrocytes in the absence (RBC) and presence of either C41 (RBC + C41 $\left.\left(0.2 \mathrm{mg} \mathrm{ml}^{-1}\right)\right)$ or $\mathrm{C} 2\left(\mathrm{RBC}+\mathrm{C} 2\left(0.2 \mathrm{mg} \mathrm{ml}^{-1}\right)\right)$. (d) Preloaded T994 merozoites were incubated with erythrocytes in the absence $(\mathrm{RBC})$ or presence of $\mathrm{mAb}$ C41 Fab fragment (C41 Fab $\left(0.2 \mathrm{mg} \mathrm{ml}^{-1}\right)$ ). The cumulative changes of $\mathrm{Ca}{ }^{2}+$ levels $\left(\sum \Delta \mathrm{F}(\mathrm{t})\right)$ in merozoites over $600 \mathrm{~s}$ were summed up and plotted against time (s). Experimental data were presented as the mean \pm s.e.m.; $n=3$. ${ }^{\star} P \leq 0.0001$ by one-way ANOVA, indicating that $C 41$ successfully blocked the $\mathrm{Ca}^{2+}$ signalling in T994 when compared with the absence of $\mathrm{C} 41 .{ }^{\#} P \leq 0.0001$ by one-way ANOVA, indicating that C41 successfully blocked the $\mathrm{Ca}^{2+}$ signalling in T994 when compared with the presence of $\mathrm{C} 2{ }^{+} P \leq 0.0001$ by one-way ANOVA, indicating that C41 Fab fragment significantly

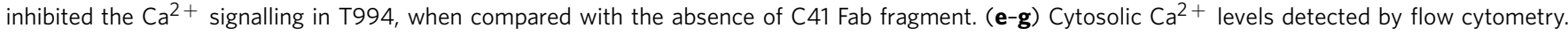
Representative FACS contour plots (e,f) were gated by size (FSC-A) versus mean fluorescence (MF) of Fluo-4 AM of merozoite (DAPI ( + )) incubated with erythrocytes in the absence (e, Positive) or presence of C41 (f, C41). Vertical gating is to separate the free merozoites with low FSC from the RBC-attached merozoites with high FSC, whereas horizontal gating is to differentiate intercellular $\mathrm{Ca}^{2}+$ levels. Q1 (green) represents free merozoites with Fluo-4 AM signals. Q2 (green) represents invading merozoites with increasing Fluo-4 AM signals. The number (blue) in Q2 represents \% population of attached merozoites. Q3 (peach) represents free merozoites with no signal. Q4 (pink) represents attached merozoites with no signal. (g) Statistical analysis of the effect of $\mathrm{C} 41$ on $\mathrm{Ca}^{2+}$ signalling during T994 merozoite invasion by FACS. C41 was able to reduce the number of invading merozoites to $56.6 \%$ (blue) and to decrease the overall $\mathrm{Ca}^{2}+$ level to $32.42 \%$ (red) during invasion, as compared with that in the absence of $\mathrm{C} 41$. Experimental data are presented as the mean \pm s.e.m.; $n=3$.

EBA175 secretion. If this is indeed correct, it would be expected that blocking EBA175 function and thereby junction formation would have no impact on $\mathrm{Ca}^{2}+$ signalling. To investigate this further, we used the invasion-blocking mAb R215, which targets EBA175 region II (ref. 30). As previously described, mAb R215 is able to dose-dependently block invasion, with maximum inhibition around $47.7 \% \pm 1.11$ at $0.25 \mathrm{mg} \mathrm{ml}^{-1}$ (Fig. 5a) $)^{30}$. However, this antibody has no effect on intracellular $\mathrm{Ca}^{2+}$ signalling during invasion (Fig. 5b, Supplementary Data 1-6).
This result confirms that invasion-blocking antibodies targeting EBA175 act after $\mathrm{Ca}^{2+}$ signalling, whereas those targeting PfRH1 act before $\mathrm{Ca}^{2}+$ signalling, and clearly highlights the different roles that are performed by PfRH1 and EBA175.

PfRH1 inhibitory mAbs block EBA175 expression. To investigate whether the inhibition of $\mathrm{Ca}^{2+}$ signalling by PfRH1 inhibitory $\mathrm{mAbs}$ has a downstream effect on the surface 

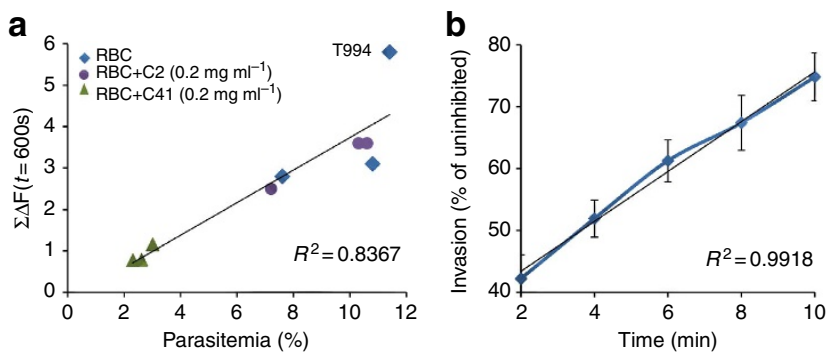

Figure 4 | Correlation between invasion and $\mathbf{C a}^{2}+$ signal. (a) T994 merozoites isolated from each $\mathrm{Ca}^{2}+$ measurement experiment (Fig. 3b) were also used in parallel to test invasion (Supplementary Table S3). The percentage of invasion (Parasitemia (\%), horizontal axis) is plotted against the $\mathrm{Ca}^{2+}$ signals at $t=600 \mathrm{~s}\left(\sum \Delta \mathrm{F}(t=600 \mathrm{~s})\right.$, vertical axis) (Fig. $\left.3 \mathrm{~b}\right)$. Blue diamonds represent merozoites incubated with erythrocytes in the absence of mAbs (RBC). Green triangles represent merozoites incubated with erythrocytes in the presence of inhibitory $\mathrm{mAb} C 41(\mathrm{RBC}+\mathrm{C} 41$ $\left(0.2 \mathrm{mg} \mathrm{ml}^{-1}\right)$ ). Purple circles represent merozoites incubated with erythrocytes in the presence of non-inhibitory $\mathrm{mAb} C 2(\mathrm{RBC}+\mathrm{C} 2$ $\left.\left(0.2 \mathrm{mg} \mathrm{ml}^{-1}\right)\right)$. The $R$-square $\left(R^{2}\right)$ with value of 0.836 indicates strong correlation between invasion and $\mathrm{Ca}^{2}+$ signalling. (b) Merozoite invasion kinetics assay under heparin treatment. Freshly isolated T994 merozoites were allowed to invade erythrocytes followed by treatment with heparin $\left(200 \mu \mathrm{g} \mathrm{ml}^{-1}\right)$ at different time points (2-10 min with $2 \mathrm{~min}$ interval) to inhibit invasion. After the final time point, cultures were incubated for $40 \mathrm{~h}$, and resulting parasitemias were analysed by flow cytometry. The proportion of merozoites that have invaded with increasing time is shown as \% of maximum invasion obtained in uninhibited culture. The rate of merozoite invasion over time is linear $\left(R^{2}=0.991\right)$. Experimental data are presented as the mean \pm s.e.m.; $n=3$.
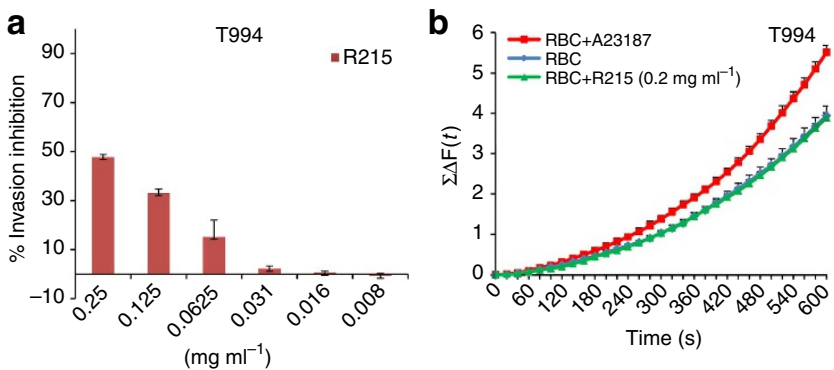

Figure 5 | The R215 mAb against EBA175 region II inhibits invasion but not $\mathbf{C a}^{2}+$ signalling. (a) Invasion inhibition assay using anti-EBA175 mAb R215 in T994 parasites. Bar charts show invasion inhibition of different concentrations $\left(0.25-0.008 \mathrm{mg} \mathrm{ml}^{-1}\right)$ of the EBA175-specific mAb R215 in T994. Bar charts show mean \pm s.e.m.; $n=3$. (b) Cytosolic $\mathrm{Ca}^{2+}$ levels were detected by using a fluorescence plate reader. Preloaded T994 merozoites with Fluo-4 AM were incubated with erythrocytes in the absence (RBC) or presence of $\mathrm{R} 215\left(\mathrm{RBC}+\mathrm{R} 215\left(0.2 \mathrm{mg} \mathrm{ml}^{-1}\right)\right) \cdot \mathrm{Ca}^{2+}$ ionophore $\mathrm{A} 23187$ $(\mathrm{RBC}+\mathrm{A} 23187)$ was used as positive control. Changes in cytosolic $\mathrm{Ca}^{2+}$ levels during merozoite invasion were assessed over time using a fluorescence plate reader. $\sum \Delta \mathrm{F}(\mathrm{t})$ that reflects the total changes of cytosolic $\mathrm{Ca}^{2}+$ levels in merozoites were plotted against time (s). Experimental data were presented as the mean \pm s.e.m.; $n=3$.

expression of EBA175, we assessed the translocation of EBA175 onto the merozoite surface. Merozoites arrested by Cyto D during erythrocyte invasion in the presence/absence of invasion inhibitory $\mathrm{mAbs}$ were surface stained with either antibodies against EBA175 or the merozoite surface marker MSP1. Surface expression of EBA175 was observed under fluorescence microscopy or measured under the fluorescence plate reader. Microneme protein EBA175 of impermeabilized merozoites will not be stained unless they are translocated to the surface of merozoites. Immunofluorescence assays clearly showed that the invasion inhibitory antibody C41 blocks surface expression of EBA175 in T994 (Fig. 6a lower panel), whereas it has no effect on T994 $\triangle \mathrm{RH} 1$ parasites (Fig. $6 \mathrm{~b}$ lower panel). In the absence of C41, EBA175 is expressed on the surface of both T994 and T994 $\Delta$ RH1 (upper panels of Fig. 6a,b). In contrast, the inhibitory antibody has no effect on the detection of MSP1 on the surface of merozoites (Fig. 6c,d). Quantification of the overall impact of EBA175 surface expression in the parasite population was assessed using a fluorescent plate reader. In T994, overall EBA175 surface expression is reduced by $>35 \%$ by the inhibitory antibody C41, whereas no impact is seen in T994ARH1 or on the surface expression of MSP1 (Fig. 6e and Supplementary Table S4). Streptolysin O (SLO) is often used to selectively permeabilize the plasma membrane of $P$. falciparum-infected erythrocytes by forming stable proteinaceous pores without altering the orientation of membrane proteins ${ }^{31}$. Permeabilization of T994 merozoites with SLO before staining with EBA175 antibody showed no difference in overall EBA175 expression (internal and surface) between free and junction-arrested merozoites in C41-treated cultures as compared with the control. This result was confirmed by performing immunofluorescence assays and by measurement using the fluorescent plate reader (Supplementary Fig. S6 and Supplementary Table S5). To further determine whether SLO could effectively permeabilize merozoite membrane to distinguish protein expression internally or on the surface, nucleoporin 100 (Nup 100; a nuclear periphery marker) and histone marker $\mathrm{H} 3 \mathrm{~K} 9 \mathrm{ac}$ were used $^{32}$. In merozoite permeabilized with SLO, Nup 100 localizes in the nuclear periphery outside the DAPI-stained area, whereas H3K9ac tends to co-localize with DAPI-stained area (Supplementary Fig. S7a,b). No staining was found in impermeabilized merozoites (Supplementary Fig. S7c,d). Taken together, these data confirm that C41 blocks EBA175 surface expression on merozoites during invasion.

We provide additional support for the surface expression of EBA175 on junction-arrested T994 merozoites using flow cytometry. Isolated merozoites were allowed to invade fresh erythrocytes in the presence of Cyto D as well as in the presence/ absence of inhibitory mAbs. In addition, labelling for EBA175 surface expression with anti-EBA175 antibodies allowed us to identify the population of free and arrested merozoites that express EBA175 on their surface. Analysis of $\sim 5,000$ DAPI $(+)$ merozoites showed that, in the absence of inhibitory antibodies, free merozoites make up $47.1 \%$ of population, of which $0.2 \%$ show EBA175 staining. In contrast, $40.7 \%$ of the $52.9 \%$ RBC-bound merozoites show EBA175 staining. In the presence of invasion inhibitory antibodies, free merozoites make up $52 \%$ of population, of which $0.5 \%$ are EBA175 positive, whereas only $17 \%$ of the $47.8 \%$ RBC-bound merozoites are EBA175 positive (Supplementary Fig. S8). The data show that there is an $\sim 45 \%$ inhibition of EBA175 surface expression in RBC-bound merozoites in the presence of the invasion inhibitory antibody (Fig. 6f), in line with the data obtained using the fluorescence plate reader (Fig. 6e). In addition, these results show that free merozoites hardly express EBA175 on the surface; furthermore, it also shows that in the absence of invasion inhibitory antibodies more RBC-attached merozoites are expressing EBA175, in line with them having formed a junction. Taken together, our data provide the first evidence that PfRH1 has a crucial role in triggering intracellular $\mathrm{Ca}^{2+}$ signal, which then leads to the subsequent release of EBA175 to form tight junction. 
a
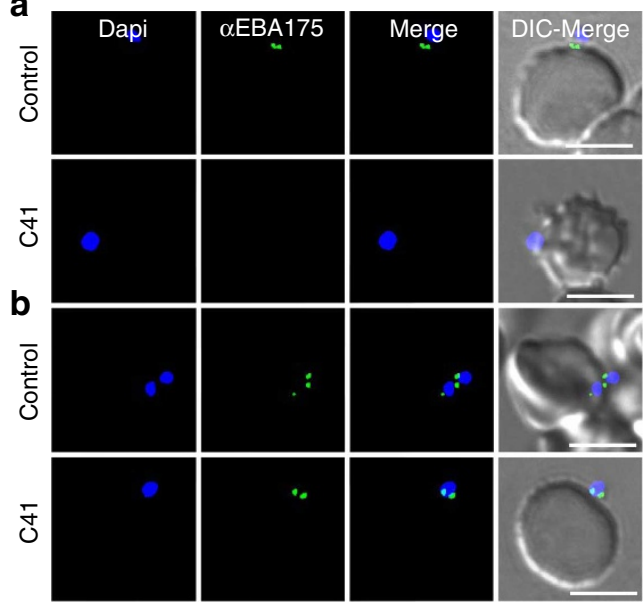

e

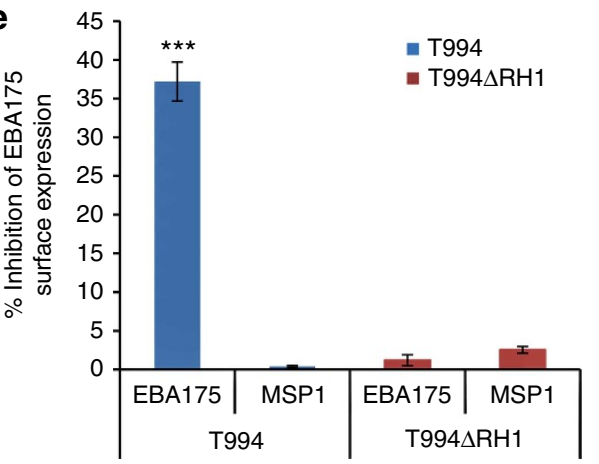

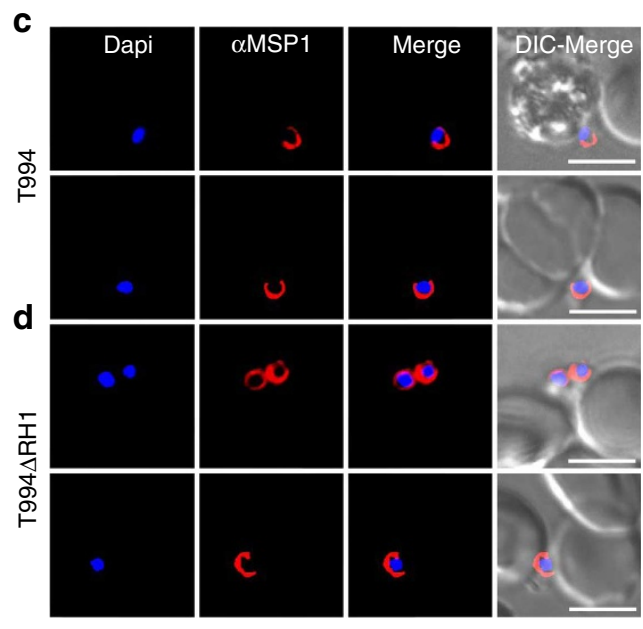

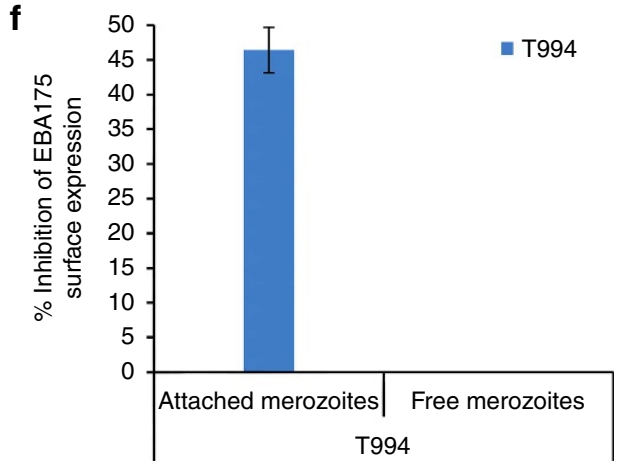

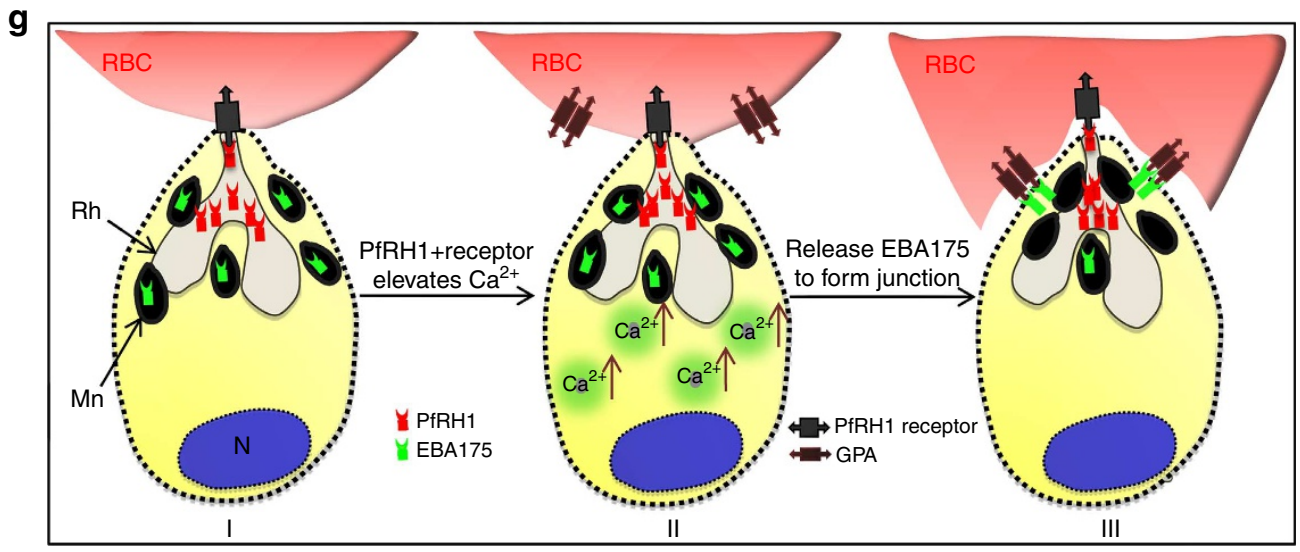

Figure 6 | Effect of anti-PfRH1 inhibitory mAbs on merozoite surface expression of EBA175 during invasion. (a-d) Surface expression of EBA175 and

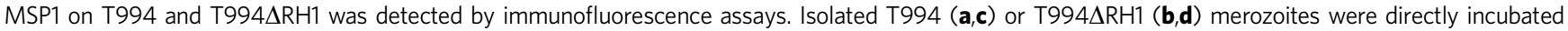
with erythrocytes containing $4 \mu \mathrm{M}$ Cyto $\mathrm{D}$ in the absence (Control) and presence (C41) of $\mathrm{C} 41\left(0.2 \mathrm{mg} \mathrm{ml}^{-1}\right)$ before analysis of expression of EBA175 and MSP1 at the junction. Both differential interference contrast (DIC) and fluorescence images were captured. Nuclear DNA was counterstained with DAPI (blue). EBA175 is shown in green, whereas MSP1 is shown in red. Fluorescence images, merged fluorescence images (DAPI and green or DAPI with red) and merged fluorescence images with DIC images are shown. Scale bars $=10 \mu \mathrm{M}$. (e) EBA175 surface expression on merozoites was detected by using a fluorescence plate reader. Surface expression of EBA175 and MSP1 on merozoite in the presence of C41 was compared with their controls. Experimental data are presented as the mean \pm s.e.m.; $n=3$. ${ }^{\star \star \star} P \leq 0.000025$ by one-way ANOVA, indicating that $C 41$ significantly decreases the surface expression of EBA175 in T994. (f) T994 merozoite EBA175 surface expression was also detected by flow cytometry during invasion (detail shown in Supplementary Fig. S8). Statistical analysis of the effect of C41 on merozoite EBA175 surface expression is shown. Merozoite attached to RBCs surface expression of EBA175 in the presence of C41 was compared with its positive control. Experimental data are presented as the mean \pm s.e.m.; $n=4$. (g) Schematic diagram of $\mathrm{Ca}^{2+}$ signalling in junction formation during erythrocyte invasion. After the merozoite initially attaches to the erythrocyte (RBC) and reorients itself, $\mathrm{PfRH} 1$ released from rhoptry (Rh) is responsible for sensing the apical end interaction (I). The binding of PfRH1 to its receptor triggers an increase of intracellular $\mathrm{Ca}^{2+}(\mathrm{II})$. Rise in cytosolic Ca ${ }^{2+}$ levels results in release of microneme (Mn) proteins such as EBA175 to bind to glycophorin A (GPA), which leads to tight junction formation (III).

\section{Discussion}

$\mathrm{Ca}^{2+}$ is a second messenger in eukaryotic cell signalling and is typically released from intracellular stores in response to an external stimulus (chemical, electrical or mechanical) that triggers a signalling cascade, which leads to a rise in cytoplasmic-free calcium $^{33}$. Apical secretion of micronemal and rhoptry proteins 
has been observed upon contact of the apicomplexan parasite Toxoplasma gondii $i^{34,35}$ as well as $P$. falciparum ${ }^{36,37}$ with their host cells, thereby mediating invasion. In T. gondii increased intracellular $\mathrm{Ca}^{2}+$ has been shown to trigger both microneme protein secretion as well as parasite egress from host cell ${ }^{35,38}$, and recently it has been shown that a rise in cytosolic $\mathrm{Ca}^{2}+$ because of the $\mathrm{Ca}^{2+}$ ionophore A23187 triggers secretion of microneme proteins such as EBA175 to the merozoite surface ${ }^{28}$. The precise signalling mechanisms that lead to the release of invasion proteins in a coordinated manner during invasion are not clear. Recent work by Singh et al. has shown that increase in the intracellular $\mathrm{Ca}^{2}+$ is the key step leading to the release of EBA175 from the microneme. EBA175 binding to its glycophorin receptor subsequently leads to the release of rhoptry proteins CLAG3.1 and PfRH2b. This is in line with our findings that PfRH1-induced $\mathrm{Ca}^{2+}$ signalling triggers EBA175 release. Previous work has shown that $\mathrm{RH}$ proteins appear to be located at the rhoptry neck, and our data would indicate that this enables at least some of the RHs to mediate the initial interactions with the erythrocyte thereby triggering $\mathrm{Ca}^{2+}$ release and microneme discharge. The bulk of the rhoptry content, including the majority of $\mathrm{RH}$, is discharged only after microneme release. It will now be interesting to establish any additional roles for RHs beyond the initial sensing. In the study by Singh et al., $\mathrm{Ca}^{2+}$ release is triggered by changing the concentration of $\mathrm{K}^{+}$in the buffer from a high to a low to reflect difference in the concentration of $\mathrm{K}^{+}$inside erythrocyte as compared with the blood plasma. In contrast, in our study, merozoites are kept in normal growth media that require PfRH1 engagement with erythrocytes to trigger $\mathrm{Ca}^{2}+$ release. In future, it is desirable to further address the effect of $\mathrm{K}^{+}$level changes compared with PfRH1-mediated changes for eliciting $\mathrm{Ca}^{2+}$ level increases. This could determine whether the $\mathrm{K}^{+}$-mediated signalling is upstream or independent of the PfRH1-mediated signalling during invasion. The relative importance of the two mechanisms in vivo also needs to be resolved. On the basis of our data, we hypothesize that the complex invasion process begins with a relatively small amount of PfRH1 exposed at the rhoptry duct interacting with its receptor on the erythrocyte surface, thereby either directly or indirectly activating a signalling cascade that leads to the release of intracellular $\mathrm{Ca}^{2}+$ stores, followed by triggering microneme and rhoptry discharge and junction formation (Fig. 6g). This is consistent with a recent finding showing that $\mathrm{Ca}^{2+}$-mediated microneme discharge is important for invasion in both T. gondii and P. falciparum ${ }^{39}$. Our results also demonstrate a function for RHs that is independent of rhoptry discharge and occurs after microneme secretion, possibly indicating that RHs are moved to an active site ahead of the release of the rest of the rhoptry content.

In future, it will now be important to further determine the mechanism by which the RH-mediated signal is passed to the merozoite to trigger $\mathrm{Ca}^{2}+$ release. Overall, our results provide new insights on the finely regulated signalling cascade that is required to ensure successful invasion of the merozoite and to open up a path for the analysis of signalling pathways involved in regulated secretion of apical organelles during invasion. Together this will provide us with a better understanding of the signalling mechanisms important in merozoite invasion and how these processes might be a new target for malaria intervention.

\section{Methods}

P. falciparum strains and culture. P. falciparum parasite lines T994, W2mef (MR4) and T994 $\mathrm{RH} 1$ (ref. 40) were cultured in fresh erythrocytes from healthy human donors (after obtaining informed consent) and RPMI supplemented with $5 \%$ albumax (Invitrogen $)^{41}$. W2mef parasites were allowed to grow in erythrocytes treated with $10 \mathrm{mU} \mathrm{ml}^{-1}$ neuraminidase (Nm; Roche). After 2-3 weeks, the
W2mef parasite adapts and grows in neuraminidase-treated erythrocytes (sialic acid independent $)^{7,26}$. This switched parasite was named W2mef/NM parasite.

PfRH1 monoclonal antibodies generation and purification. PfRH1 hybridoma cells were developed by BioGenes (Germany) using purified soluble recombinant protein PfRH1-RII-3 (ref. 7). Positively selected hybridoma cells were cultured in 2\% FBS-DMEM (Invitrogen). Purification of PfRH1 mAbs IgG from cell culture medium was carried out by using standard purification procedures (HiTrap Protein G HP, GE healthcare). Monoclonal antibody C41 Fab fragment was generated and purified by using standard procedures (Pierce Fab Micro Preparation Kit, Thermo Scientific). The authenticity of the Fab fragment was confirmed by $12 \%$ SDS-PAGE (Bio-Rad) followed by coomassie blue staining (Sigma), which yielded one band of $\sim 50 \mathrm{kDa}$ under nonreducing conditions.

Western blot of parasite extracts and culture supernatants. Parasite culture was synchronized at the ring stage with $5 \%$ D-sorbitol (Sigma) and cultured to mature schizont stages ${ }^{42,43}$. Tightly synchronized late schizonts were purified using $68 \%$ Percoll gradient centrifugation ${ }^{44}$. Merozoites were purified from schizont extract by filtration under nitrogen cavitation ${ }^{45-48}$. Schizonts and merozoite extracts were washed twice with PBS, lysed directly into sample buffer and frozen and thawed three times, followed by separation on $12 \%$ SDS-PAGE. Mouse mAb g12 $(1: 1,000, \text { MR4 })^{49}$ was used as a loading control for merozoite extracts, whereas rabbit $\alpha$-actin polyclonal antibodies (1:1,000, Sigma) were used for schizont extracts. To make parasite culture supernatant, purified schizonts were allowed to grow in the absence of RBC at $37^{\circ} \mathrm{C}$ with shaking. Cells were collected by centrifugation after $16 \mathrm{~h}$, and supernatants were either stored in aliquots at $-80^{\circ} \mathrm{C}$ or directly separated on 6\% SDS-PAGE. Micronemal marker EBA175-specific antibody ${ }^{50}(1: 5,000$, MR4) was a loading control for parasite culture supernatant. Following electrophoresis, gels were transferred onto nitrocellulose membranes $(0.2 \mu \mathrm{m})$ (Bio-Rad). Specific proteins were detected by using PfRH1 mAbs $(1: 5,000)$, followed by HRP-linked secondary antibodies $(1: 10,000)$ and enhanced chemiluminescence (GE healthcare).

Invasion inhibition assay. Synchronized late-stage schizonts were purified, and $160 \mu \mathrm{l}$ of parasite suspension was added in duplicate in a 96-well flat bottom microtitre plate containing PfRH1 mAbs $\left(0.008-0.5 \mathrm{mg} \mathrm{ml}^{-1}\right)$, purified C41 Fab fragment $\left(0.008-0.5 \mathrm{mg} \mathrm{ml}^{-1}\right)$ or EBA175 mAb R215 (0.008-0.25 $\left.\mathrm{mg} \mathrm{ml}^{-1}\right)$, respectively. A total of 1,000 erythrocytes were scored for presence of rings on Giemsa-stained smears after $24 \mathrm{~h}$ for reinvasion. Invasion (Inv) was assessed as (\%) parasitemia. (\%) Parasitemia $=$ (total number of ring-infected RBCs/total number of RBCs $) \times 100$. Invasion in the presence of mAbs was compared with positive controls of invasion of the same parasite lines in normal complete RPMI. Invasion inhibition efficiencies were determined as follows: (\%) inhibition efficiency = $\left(1-\operatorname{Inv}(\mathrm{mAbs})_{\text {dilu }} / \operatorname{Inv}(\right.$ positive $\left.)\right) \times 100$. Data shown are from three biological replicates. Each biological replicate has two technical replicates. Experimental data are presented as the mean \pm s.e.m.

Erythrocyte binding assays. Hundred microliters of T994 parasite culture supernatant was incubated with $100 \mu \mathrm{l}$ of packed normal erythrocytes in the presence of PfRH1 mAbs (at final concentration ranging from 0.3 down to $\left.0.035 \mathrm{mg} \mathrm{ml}^{-1}\right)$ at $37^{\circ} \mathrm{C}$ for $1 \mathrm{~h}^{7,26}$.

Junction merozoite blocking assay. Merozoites were arrested in the erythrocyte junction by treatment with cytochalasin D (Cyto D) (Sigma) ${ }^{26,27}$. Purified latestage schizont parasites were added to the completed RPMI containing fresh erythrocytes in 96-well plates. Cyto D was added to the final concentration of $0.1 \mu \mathrm{M}$. PfRH1 mAbs were also present at $0.2 \mathrm{mg} \mathrm{ml}^{-1}$ concentration if required. After $8 \mathrm{~h}$ incubation, Giemsa (Sigma)-stained smears were prepared for counting merozoites arrested at junction formation by using light microscopy. A total of 1,000 erythrocytes were scored. The number of junction-arrested merozoites in the presence of mAbs was compared with that in a positive control of the same parasite. Junction merozoite inhibition efficiencies were determined as follows: $\%$ inhibition of junction merozoite $=(1-$ total number of junction merozoites $(\mathrm{mAbs}) /$ total number of junction merozoites (positive control)) $\times 100$. Data shown are from three biological replicates. Each biological replicate has two technical replicates. Experimental data are presented as the mean \pm s.e.m.

Expression of four overlapping regions from RII-3. Four N-terminal overlapping fragments from RII-3 were amplified by PCR, expressed as His-tag recombinant proteins by BL21 (DE3) (Stratagene) ${ }^{7}$, named N1 (500-692 amino acid (aa)), N2 (500-726 aa), N3 (500-746 aa) and N4 (500-773 aa), followed by $12 \%$ SDS-PAGE and probed with mAbs C49, C41, C2 and C50, respectively $(1: 5,000)$. A mAb against $6 \mathrm{X}$ His-tag ( $\alpha$ His, 1:5,000, Clonetech) was used as loading control. For the PCR, the forward primer was $5^{\prime}$-GACCATATGTTACAAATAG TACAACAAAAACTTTTAGAAATC- $3^{\prime}$ and the reverse primers were $5^{\prime}$-AACCT CGAGAGAAATAAGTTGAATCGTCTCATTATTTT-3' (N1), 5' -AACCTCGA GGGATTTAAGAAGATTTTGGATGTTTT-3' (N2), 5' -AACCTCGAGTTGT 
TTTAATATATATTTAGAAATTGTGTCTATGAA-3' (N3), and 5'-AACCTC GAGGTTTTGTTCATTTTTTATTTCTTCAAGAT-3' (N4).

Peptide generation and epitope mapping of PfRH1. Peptide design was based on the mAbs recognition of four His-tag recombinant overlapping fragments derived from RII-3 by western blot (Supplementary Fig. S3a-e). All biotinylated overlapping synthetic peptides were synthesized by Peptide Synthesis Core facilities, SBS, NTU. The lyophilized peptides were dissolved in dimethyl sulfoxide (DMSO, Sigma). Peptide stocks were diluted in PBS $+0.1 \%$ BSA to make up $2.5 \mu \mathrm{g} \mathrm{ml}^{-1}$. The streptavidin-coated 96-well plate, pre-blocked with Superblock blocking buffer (Thermo Scientific), was washed three times with $200 \mu \mathrm{l}$ wash buffer $(25 \mathrm{mM}$ Tris, $150 \mathrm{mM} \mathrm{NaCl}, \mathrm{pH} 7.2,0.1 \% \mathrm{BSA}, 0.05 \%$ Tween 20 ). Hundred microliters of the peptide solution was added into each well of the streptavidin-coated plate and incubated for $2 \mathrm{~h}$ at room temperature (RT) with gentle shaking. After four washes, $100 \mu \mathrm{l}$ of mAb (C49, C41, C2 or C50 in $25 \mathrm{mM}$ Tris, $150 \mathrm{mM} \mathrm{NaCl}, \mathrm{pH}$ 7.2, $0.1 \%$ BSA) at a concentration of $2.5 \mu \mathrm{g} \mathrm{ml}^{-1}$ was added into each well and incubated for $1 \mathrm{~h}$ at RT. The peptide was washed four times followed by the addition of $100 \mu \mathrm{l}$ of HRP goat anti-mouse IgG (1:3,000; Biolegend). After six washes, $50 \mu \mathrm{l}$ of 3,3',5,5'Tetramethylbenzidine (TMB) high sensitive substrate solution (Biolegend) was added into each well. The plates were incubated for 3-5 min before analysing by using a Benchmark PlusMicroplate Spectrophotometer (BioRad Laboratories Inc) at $370 \mathrm{~nm}$.

Live video microscopy. For live video microscopy ${ }^{23}$, we used an Axiovert $200 \mathrm{M}$ cell observer with a $37^{\circ} \mathrm{C}$ chamber with a continuous supply of $5 \% \mathrm{CO}_{2}$. Five to ten percentage of the late-schizont stage parasites were diluted at 1:100 in complete RPMI. Live video was performed using $35 \mathrm{~mm}$ glass bottom dishes. The video was captured in the presence or absence of PfRH1 mAbs (C2, C41, C49 and C50) at a final concentration of $0.2 \mathrm{mg} \mathrm{ml}^{-1}$. AxioCam $\mathrm{HRm}$ camera and Axiovision software, or Cool SNAP camera and MetaMorph software, were used for image acquisition. The video files were prepared using Image J.

Isolation of $\boldsymbol{P}$. falciparum merozoites. Purified $P$. falciparum schizonts were resuspended in warm complete RPMI and allowed to rupture and release merozoites over a period of $2 \mathrm{~h}$ at $37^{\circ} \mathrm{C}$. Cultures containing unruptured schizonts and released merozoites were centrifuged at 2,200 r.p.m. ( $942 \mathrm{~g}$ ) on an Eppendorf 5810R centrifuge for $3 \mathrm{~min}$ to separate released merozoites from unruptured schizonts and uninfected erythrocytes. Supernatants containing free merozoites were then centrifuged at 5,000 r.p.m. (4,500 g, Sigma 3K15) for $3 \mathrm{~min}$ to collect merozoites. The merozoites were immediately resuspended in warm complete RPMI medium for further studies.

Viability assay of free merozoites. Erythrocyte invasion assays were performed in parallel to assess the viability of isolated free merozoites tested for $\mathrm{Ca}^{2+}$ measurement. After $24-30 \mathrm{~h}$ post-invasion, newly invaded ring-infected erythrocytes were scored by Giemsa (Sigma, USA) staining. Invasion rates were determined as (\%) parasitemia. Data were shown from three biological replicates.

Kinetics of merozoite invasion by heparin treatment. To define the kinetics of merozoite invasion, the invasion inhibitor heparin was used to block merozoite invasion $^{29}$. In brief, freshly isolated T994 merozoites were allowed to invade erythrocytes in the 96-well plate followed by addition of heparin $\left(200 \mu \mathrm{g} \mathrm{ml}^{-1}\right)$ at different time points (2-10 min with $2 \mathrm{~min}$ interval) to inhibit further invasion. After the final time point, cultures were incubated at $37^{\circ} \mathrm{C}$ for $40 \mathrm{~h}$. The plate was centrifuged at 2,200 r.p.m. ( $942 \mathrm{~g}$ ) for $3 \mathrm{~min}$ to separate free merozoites from uninfected and infected erythrocytes. The culture was washed with complete RPMI twice and stained with $40 \mathrm{nM} \mathrm{4}$,6-diamidino-2-phenylindole (DAPI, Molecular Probes, USA). The resulting parasitemias were analysed by flow cytometry. The cells were first gated by size in SSC/FSC, followed by further gating in FSC-A/FSC$\mathrm{H}$ to discriminate doublets from single cells. Uninfected erythrocytes were gated as baseline by DAPI fluorescence in FSC-A/DAPI-A. The proportion of merozoites that have invaded with increasing time is shown as $\%$ of maximum invasion obtained in uninhibited culture. Data shown were from three biological replicates. Experimental data were presented as the mean \pm s.e.m.; $n=3$.

Real-time $\mathrm{Ca}^{2+}$ measurement in merozoites during invasion. Fresh isolated merozoites were preloaded with $6 \mu \mathrm{M}$ Fluo-4 AM (Invitrogen) in complete RPMI at $37^{\circ} \mathrm{C}$ for $20 \mathrm{~min}$. Preloaded merozoites were washed twice with warm complete RPMI and then immediately resuspended in fresh erythrocytes or in the presence of $4 \mu \mathrm{M}$ Cyto D, C41, C2, C41 Fab fragment or EBA175 mAbs R215 at $0.2 \mathrm{mg} \mathrm{ml}^{-1}$, or $10 \mu \mathrm{M} \mathrm{Ca}^{2+}$ ionophore A23187 (Calbiochem, USA), or pretreated with $50 \mu \mathrm{M} \mathrm{Ca}^{2+}$ chelator BAPTA-AM (Calbiochem, USA) followed by A23187 (ref. 28), and cultured in Greiner 96 Black Flat Bottom Polystyrol plates. Changes in cytosolic $\mathrm{Ca}^{2+}$ levels during merozoite invasion were timely assessed using a fluorescence plate reader (Infinite M200; Tecan). Fluorescence intensity was measured for $600 \mathrm{~s}$ with excitation at $488 \mathrm{~nm}$ and emission at $530 \mathrm{~nm}$ by TECAN- $i$ control 1.8.20.0 software. The maximum increase of $\mathrm{Ca}^{2+}$ green fluorescence $(\mathrm{F})$ during a period of $600 \mathrm{~s}$ after the start of the stimulation was normalized to the fluorescence intensity before stimulation $\left(\mathrm{F}_{0}\right)$. Changes in cytosolic $\mathrm{Ca}^{2+}$ levels $(\Delta \mathrm{F}(\mathrm{t}))$ were expressed as changes in fluorescence intensity as a percentage of the starting fluorescence levels: $\Delta \mathrm{F}(\mathrm{t})=\left(\mathrm{F}-\mathrm{F}_{0}\right) / \mathrm{F}_{0}$ where $\mathrm{F}=$ fluorescence intensity $(\mathrm{t}), \mathrm{F}_{0}=$ starting fluorescence signal. The total changes of $\mathrm{Ca}^{2+}$ levels $\left(\sum \Delta \mathrm{F}(\mathrm{t})\right)$ in merozoites along the $600 \mathrm{~s}$ were summed up and plotted against time (s). Data shown were from three biological replicates. Experimental data were presented as the mean \pm s.e.m. TECAN scanning was performed at $37^{\circ} \mathrm{C}$.

\section{Flow cytometry $\mathrm{Ca}^{2}+$ measurement in merozoites during invasion. Isolated}

T994 merozoites were preloaded with Fluo-4 AM as well as labelled with DAPI as described above, followed by incubation with fresh warm erythrocytes containing $4 \mu \mathrm{M}$ Cyto D in the absence and presence of $\mathrm{mAb} C 41\left(0.2 \mathrm{mg} \mathrm{ml}^{-1}\right)$. The culture was immediately processed using a BD LSR II flow cytometer (BD Biosciences). Merozoites that are invading will be arrested by Cyto D after junction formation, whereas merozoites in the presence of inhibitory antibodies will either detach or remain attached to the RBC without forming a junction. Each culture was excited with light at 488 and $355 \mathrm{~nm}$, and fluorescence signal was detected with a 530/30 and $440 / 40 \mathrm{~nm}$ band pass filters. In each experiment, data were acquired for $\sim 20,000$ DAPI $(+)$ merozoites with the same settings for each condition. A histogram representing two distinct cell populations among these 20,000 cells indicates free merozoites and RBC-bound merozoites. Preloaded merozoites alone were measured as the baseline for detection of Fluo-4 AM signals. The cells were gated by FSC-A versus Fluo-4 AM mean fluorescence (MF). We observed the $\%$ parent population of attached merozoites with increasing $\mathrm{Ca}^{2+}$ signal from the culture in the absence (positive) or presence of $\mathrm{C} 41$ (C41) and investigated the effect of $\mathrm{C} 41$ on signalling during invasion. Data were analysed by using $\mathrm{BD}$ FASCDiva software. (\%) Inhibition of attached merozoites $=(1-\%$ parent population $(\mathrm{C} 41) / \%$ parent population (positive) $) \times 100$. (\%) Decrease of Fluo-4 $\mathrm{AM}$ mean fluorescence $(\mathrm{MF})=(1-\mathrm{MF}(\mathrm{C} 41) / \mathrm{MF}($ positive $)) \times 100$. Data shown are from three biological replicates. Experimental data are presented as the mean \pm s.e.m.

Measurement of intracellular $\mathbf{C a}^{2}+$ levels on free merozoites. Merozoites preloaded with Fluo-4 AM as described above were immediately resuspended in complete RPMI only or treated with $10 \mu \mathrm{M} \mathrm{A} 23187$ for $15 \mathrm{~min}$ at $37^{\circ} \mathrm{C}$ with or without pretreatment with $50 \mu \mathrm{M}$ BAPTA-AM for $15 \mathrm{~min}$ at $37^{\circ} \mathrm{C}$ (ref. 28). Changes in cytosolic $\mathrm{Ca}^{2+}$ levels were assessed using flow cytometry. Preloaded merozoites in complete RPMI were measured as baselines. Merozoites were excited at $488 \mathrm{~nm}$, and fluorescence signal is detected with a $530 / 30 \mathrm{~nm}$ band pass filter for periods of $2 \mathrm{~min}$ on BD LSR II (BD Biosciences). Merozoites were gated on the basis of their forward scatter (FSC) and side scatter (SSC). Data were evaluated by using dot plot of Fluo-4 AM mean fluorescence (MF) versus time using BD FACSDiva software to visualize the cytosolic $\mathrm{Ca}^{2}+$ levels in merozoites. The effect of BAPTA-AM ( $\left.\mathrm{MF}_{\text {BAPTA-AM}}\right)$ on free merozoites $\mathrm{Ca}^{2+}$ signalling was compared with that of $A 23187\left(\mathrm{MF}_{\mathrm{A} 23187}\right)$. (\%) Inhibition of BAPTA-AM $=\left(1-\left(\mathrm{MF}_{\mathrm{BAPTA}}\right.\right.$ $\left.\left.\mathrm{AM} / \mathrm{MF}_{\mathrm{A} 23187}\right)\right) \times 100$. Data shown are from three biological replicates.

Experimental data are presented as the mean \pm s.e.m.

Immunofluorescence assays and microscopy. Synchronized late-stage schizonts were smeared and air-dried followed by fixation with acetone (Merck) for $5 \mathrm{~min}$ at RT. Smears were incubated with PfRH1 mAbs (1:800) and co-incubated with rabbit $\alpha$-EBA175 (1:800) for $1 \mathrm{~h}$ in 3\% BSA/PBS buffer, followed by three times for $5 \mathrm{~min}$ washes in $1 \times$ PBS. They were then incubated with a mixture of Alex Flour 594 goat anti-mouse IgG $(\mathrm{H}+\mathrm{L})$ and Alex Flour 488 goat anti-rabbit $\operatorname{IgG}(\mathrm{H}+\mathrm{L})$ secondary antibodies (1:1,000, respectively, Molecular Probe) for $1 \mathrm{~h}$. Slides were washed in $1 \times$ PBS for three times for 5 min. Slides were air-dried, mounting medium containing DAPI was applied (Vector laboratories), and the coverslips were sealed. The fluorescence images were captured using a LSM510 Confocal Microscope (Carl Zeiss).

Immunofluorescence assay for merozoites. Isolated T994 merozoites were incubated with or without streptolysin O (SLO, Sigma) $)^{51}$. The culture was washed three times with incomplete RPMI and then fixed with $1 \%$ p-formaldehyde (Sigma) for $45 \mathrm{~min}$ at RT followed by centrifugation at 5,000 r.p.m. (4,500 g, Sigma 3K15) for $3 \mathrm{~min}$. After two washes, the culture was incubated with either rabbit $\alpha$-Nucleoporin 100 ( $\alpha$-Nup 100, 1:500), a nuclear periphery marker, or rabbit $\alpha$-acetyl-Histone H3 (Lys9) antibody ( $\alpha$-H3K9ac, 1:500, Millipore), a histone marker ${ }^{32}$ for $45 \mathrm{~min}$ at $37^{\circ} \mathrm{C}$. The culture was washed three times in incomplete RPMI followed by incubation with Alex Flour 594 goat anti-rabbit IgG $(\mathrm{H}+\mathrm{L})$ $(1: 1,000)$ or Alex Flour 488 goat anti-rabbit IgG $(\mathrm{H}+\mathrm{L})(1: 1,000)$ together with DAPI for $45 \mathrm{~min}$ at RT. The culture was washed three times and then applied to the slides. The images were captured by Nikon TE2000-U Inverted Fluorescence Microscopy.

Detection of EBA175 on merozoite surface by fluorescence-based methods. Isolated merozoites from T994 and T994 $\Delta$ RH1 were incubated with fresh erythrocytes containing $4 \mu \mathrm{M}$ Cyto D in the absence and presence of $\mathrm{mAb}$ C41 
$\left(0.2 \mathrm{mg} \mathrm{ml}^{-1}\right)$ at $37^{\circ} \mathrm{C}$ for $20 \mathrm{~min}$ followed by incubation with or without Streptolysin O (SLO, Sigma $)^{51}$. The culture was washed three times with $1 \%$ BSA/PBS and then fixed with $1 \%$ p-formaldehyde (Sigma) for $45 \mathrm{~min}$ at RT followed by centrifugation at 5,000 r.p.m. (4,500 g, Sigma 3K15) for 3 min. After two washes, the culture was incubated with rabbit $\alpha$-EBA175 (1:500) for $45 \mathrm{~min}$ at $37^{\circ} \mathrm{C}$. The culture was washed three times with incomplete RPMI followed by incubation with Alex Flour 488 goat anti-rabbit IgG $(\mathrm{H}+\mathrm{L})(1: 1,000)$ together with DAPI for $45 \mathrm{~min}$ at RT. The culture was washed three times with incomplete RPMI. The culture was finally resuspended in $100 \mu \mathrm{l}$ complete RPMI and transferred into Greiner 96 Black Flat Bottom Polystyrol plates for analysis by using a fluorescence plate reader using the program TECAN-i-control 1.8.20.0. The duplicated culture (except SLO treated culture) was also tested using rabbit $\alpha$-MSP1 (1:1,000, MR4) followed by incubation with Alex Flour 594 goat anti-rabbit IgG $(\mathrm{H}+\mathrm{L})(1: 1,000)$ together with DAPI. The samples were applied to the slides. The images were captured by Nikon TE2000-U Inverted Fluorescence Microscopy. To further confirm and quantify the effect of inhibitory mAbs C41 on the surface expression of EBA175, fluorescence intensity was also measured by using a fluorescence plate reader. Fluorescence signal of EBA175 $\left(\mathrm{F}_{\mathrm{EBA} 175}\right)$ was excited at $488 \mathrm{~nm}$ and emitted at $519 \mathrm{~nm}, \mathrm{MSP} 1$ surface staining $\left(\mathrm{F}_{\mathrm{MSP} 1}\right)$ was excited at $590 \mathrm{~nm}$ and emitted at $617 \mathrm{~nm}$ and nuclear signal ( $\mathrm{F}_{\text {EBA175-DAPI }}$ or $\left.\mathrm{F}_{\mathrm{MSP1} \text {-DAPI }}\right)$ was excited at $375 \mathrm{~nm}$ and emitted at $461 \mathrm{~nm}$. Fluorescence signal was normalized to nuclear DAPI signal as follows: $\mathrm{EBA} 175$ ratio $=\mathrm{F}_{\mathrm{EBA} 175} / \mathrm{F}_{\mathrm{EBA} 175-\mathrm{DAPI}}$ and $\mathrm{MSP} 1$ ratio $=\mathrm{F}_{\mathrm{MSP1} 1} / \mathrm{F}_{\mathrm{MSP} 1-\mathrm{DAPI}}$. Protein expression on merozoite surface in the presence of mAbs C41 was compared with its control of the same culture incubated in the absence of C41. Surface expression inhibition efficiencies were determined as follows: (\%) inhibition of EBA175 surface expression $=(1-\mathrm{EBA} 175$ ratio $(\mathrm{C} 41) / \mathrm{EBA} 175$ ratio $($ control) $) \times 100$ and $(\%)$ inhibition of MSP1 surface expression $=(1-$ MSP1 ratio $($ C41)/MSP1 ratio $($ control)) $\times 100$. Protein expression on merozoite permeabilized with SLO in the presence of mAbs C41 were compared with its control of the same culture in the absence of C41. EBA175 expression inhibition efficiencies were determined as follows: (\%) inhibition of EBA175 expression $=(1-$ EBA175 ratio $(\mathrm{C} 41-\mathrm{SLO}) / \mathrm{EBA} 175$ ratio $($ control $-\mathrm{SLO}))$ $\times 100$. Data shown are from three biological replicates. Experimental data are presented as the mean \pm s.e.m.

Detection of EBA175 on merozoite surface by flow cytometry. The culture prepared for detection of EBA175 by fluorescence microscopy, mentioned above, was resuspended in complete RPMI and used for analysis by BD LSR II (BD Biosciences). In each experiment, data were acquired for 5,000 DAPI $(+)$ merozoites with the same settings for each condition. A histogram representing two distinct cell populations among these 5,000 cells indicates free merozoites (Free Mero) and RBC-bound merozoites (Attached Mero + RBCs). We observed these two populations individually and investigated the effect of C41 on EBA175 surface expression in T994. Each culture was excited with light at 488 and $355 \mathrm{~nm}$, and fluorescence signal was detected with $530 / 30$ and $440 / 40 \mathrm{~nm}$ band pass filters. The cells were gated on the basis of their FSC and SSC. Data were analysed using BD FASCDiva software. Histograms showing logarithmic green fluorescence intensities (FITC-A) versus blue fluorescence intensities (DAPI-A) were plotted. For each experiment, fixed untreated cultures were stained only with FITC-conjugated antirabbit IgG to establish background staining and to set up a gate before calculating the percentage of FITC $(+)$ merozoites (that is, those expressing EBA175 on their surface) in the parent population. EBA175 surface expression on junction-arrested merozoites in the presence of $\mathrm{mAb} C 41$ was compared with a positive control in the absence of C41. EBA175 surface expression inhibition efficiencies were determined as follows: (\%) inhibition of EBA175 surface expression $=(1-\%$ parent population $(\mathrm{C} 41) / \%$ parent population (positive) $\times 100$. Data shown are from four biological replicates. Experimental data are presented as the mean \pm s.e.m.

Statistical analysis. Statistical comparison was done using one-way ANOVA as appropriate. The significance level was set at $P<0.05$. The $P$-value is provided for some individual experiments.

Raw data of absolute measurement $\mathbf{C a}^{\mathbf{2}}+$ signalling. Absolute measurement raw data of all $\mathrm{Ca}^{2+}$ signalling experiments have been provided in Supplementary Data 1-6.

\section{References}

1. Cowman, A. F. \& Crabb, B. S. Invasion of red blood cells by malaria parasites. Cell 124, 755-766 (2006).

2. Baum, J., Gilberger, T. W., Frischknecht, F. \& Meissner, M. Host-cell invasion by malaria parasites: insights from Plasmodium and Toxoplasma. Trends Parasitol. 24, 557-563 (2008).

3. Gaur, D. \& Chitnis, C. E. Molecular interactions and signalling mechanisms during erythrocyte invasion by malaria parasites. Curr. Opin. Microbiol. 14, 422-428 (2011).

4. Gunalan, K., Gao, X., Yap, S. S., Huang, X. \& Preiser, P. R. The role of the reticulocyte-binding-like protein homologues of Plasmodium in erythrocyte sensing and invasion. Cell Microbiol. 15, 35-44 (2013).
5. Chitnis, C. E. \& Miller, L. H. Identification of the erythrocyte binding domains of Plasmodium vivax and Plasmodium knowlesi proteins involved in erythrocyte invasion. J. Exp. Med. 180, 497-506 (1994).

6. Baum, J. et al. Reticulocyte-binding protein homologue 5 - an essential adhesin involved in invasion of human erythrocytes by Plasmodium falciparum. Int. J. Parasitol. 39, 371-380 (2009).

7. Gao, X. et al. Antibodies targeting the PfRH1 binding domain inhibit invasion of Plasmodium falciparum merozoites. PLoS Pathog 4, e1000104 (2008).

8. Gaur, D., Singh, S., Jiang, L., Diouf, A. \& Miller, L. H. Recombinant Plasmodium falciparum reticulocyte homology protein 4 binds to erythrocytes and blocks invasion. Proc. Natl Acad. Sci. USA 104, 17789-17794 (2007).

9. Rayner, J. C., Vargas-Serrato, E., Huber, C. S., Galinski, M. R. \& Barnwell, J. W. A Plasmodium falciparum homologue of Plasmodium vivax reticulocyte binding protein (PvRBP1) defines a trypsin-resistant erythrocyte invasion pathway. J. Exp. Med. 194, 1571-1581 (2001).

10. Sahar, T. et al. Plasmodium falciparum reticulocyte binding-like homologue protein 2 (PfRH2) is a key adhesive molecule involved in erythrocyte invasion. PLoS One 6, el7102 (2011).

11. Tham, W. H. et al. Antibodies to reticulocyte binding protein-like homologue 4 inhibit invasion of Plasmodium falciparum into human erythrocytes. Infect. Immun. 77, 2427-2435 (2009).

12. Triglia, T. et al. Identification of proteins from Plasmodium falciparum that are homologous to reticulocyte binding proteins in Plasmodium vivax. Infect. Immun. 69, 1084-1092 (2001).

13. Rodriguez, M., Lustigman, S., Montero, E., Oksov, Y. \& Lobo, C. A. PfRH5: a novel reticulocyte-binding family homolog of Plasmodium falciparum that binds to the erythrocyte, and an investigation of its receptor. PLoS One 3, e3300 (2008).

14. Tham, W. H. et al. Complement receptor 1 is the host erythrocyte receptor for Plasmodium falciparum PfRh4 invasion ligand. Proc. Natl Acad. Sci. USA 107, 17327-17332 (2010).

15. Crosnier, C. et al. Basigin is a receptor essential for erythrocyte invasion by Plasmodium falciparum. Nature 480, 534-537 (2011).

16. Galinski, M. R., Medina, C. C., Ingravallo, P. \& Barnwell, J. W. A reticulocytebinding protein complex of Plasmodium vivax merozoites. Cell 69, 1213-1226 (1992).

17. Galinski, M. R., Xu, M. \& Barnwell, J. W. Plasmodium vivax reticulocyte binding protein-2 (PvRBP-2) shares structural features with PvRBP-1 and the Plasmodium yoelii $235 \mathrm{kDa}$ rhoptry protein family. Mol. Biochem. Parasitol. 108, 257-262 (2000).

18. Singh, A. P. et al. Targeted deletion of Plasmodium knowlesi Duffy binding protein confirms its role in junction formation during invasion. Mol. Microbiol. 55, 1925-1934 (2005).

19. Stubbs, J. et al. Molecular mechanism for switching of $P$. falciparum invasion pathways into human erythrocytes. Science 309, 1384-1387 (2005).

20. Chopineau, M., Maurel, M. C., Combarnous, Y. \& Durand, P. Topography of equine chorionic gonadotropin epitopes relative to the luteinizing hormone and follicle-stimulating hormone receptor interaction sites. Mol. Cell Endocrinol. 92, 229-239 (1993).

21. Imakiire, T. et al. Generation, immunologic characterization and antitumor effects of human monoclonal antibodies for carcinoembryonic antigen. Int. J. Cancer 108, 564-570 (2004).

22. Martin, P. J. et al. Identification and functional characterization of two distinct epitopes on the human $\mathrm{T}$ cell surface protein Tp50. J. Immunol. 131, 180-185 (1983).

23. Treeck, M. et al. Functional analysis of the leading malaria vaccine candidate AMA-1 reveals an essential role for the cytoplasmic domain in the invasion process. PLoS Pathog. 5, el000322 (2009).

24. Triglia, T., Tham, W. H., Hodder, A. \& Cowman, A. F. Reticulocyte binding protein homologues are key adhesins during erythrocyte invasion by Plasmodium falciparum. Cell Microbiol. 11, 1671-1687 (2009).

25. Triglia, T. et al. Plasmodium falciparum merozoite invasion is inhibited by antibodies that target the PfRh2a and b binding domains. PLoS Pathog. 7, e1002075 (2011).

26. Gunalan, K., Gao, X., Liew, K. J. \& Preiser, P. R. Differences in erythrocyte receptor specificity of different parts of the Plasmodium falciparum reticulocyte binding protein homologue 2a. Infect. Immun. 79, 3421-3430 (2011).

27. Miller, L. H., Aikawa, M., Johnson, J. G. \& Shiroishi, T. Interaction between cytochalasin B-treated malarial parasites and erythrocytes. Attachment and junction formation. J. Exp. Med. 149, 172-184 (1979).

28. Singh, S., Alam, M. M., Pal-Bhowmick, I., Brzostowski, J. A. \& Chitnis, C. E. Distinct external signals trigger sequential release of apical organelles during erythrocyte invasion by malaria parasites. PLoS Pathog. 6, e1000746 (2010).

29. Boyle, M. J. et al. Isolation of viable Plasmodium falciparum merozoites to define erythrocyte invasion events and advance vaccine and drug development. Proc. Natl Acad. Sci. USA 107, 14378-14383 (2010). 
30. Sim, B. K. et al. Delineation of stage specific expression of Plasmodium falciparum EBA-175 by biologically functional region II monoclonal antibodies. PloS One 6, e18393 (2011).

31. Ansorge, I., Benting, J., Bhakdi, S. \& Lingelbach, K. Protein sorting in Plasmodium falciparum-infected red blood cells permeabilized with the poreforming protein streptolysin O. Biochem. J. 315(Pt 1): 307-314 (1996).

32. Volz, J. et al. Potential epigenetic regulatory proteins localise to distinct nuclear sub-compartments in Plasmodium falciparum. Int. J. Parasitol. 40, 109-121 (2010).

33. Erxleben, C. et al. Microdomain Ca2 + activation during exocytosis in Paramecium cells. Superposition of local subplasmalemmal calcium store activation by local Ca2 + influx. J. Cell Biol. 136, 597-607 (1997).

34. Carruthers, V. B. \& Sibley, L. D. Sequential protein secretion from three distinct organelles of Toxoplasma gondii accompanies invasion of human fibroblasts. Eur. J. Cell Biol. 73, 114-123 (1997).

35. Carruthers, V. B. \& Sibley, L. D. Mobilization of intracellular calcium stimulates microneme discharge in Toxoplasma gondii. Mol. Microbiol. 31, 421-428 (1999).

36. Hakansson, S., Charron, A. J. \& Sibley, L. D. Toxoplasma evacuoles: a two-step process of secretion and fusion forms the parasitophorous vacuole. $E M B O J .20$, 3132-3144 (2001)

37. Besteiro, S., Michelin, A., Poncet, J., Dubremetz, J. F. \& Lebrun, M. Export of a Toxoplasma gondii rhoptry neck protein complex at the host cell membrane to form the moving junction during invasion. PLoS Pathog. 5, e1000309 (2009).

38. Lovett, J. L. \& Sibley, L. D. Intracellular calcium stores in Toxoplasma gondii govern invasion of host cells. J. Cell Sci. 116, 3009-3016 (2003).

39. Farrell, A. et al. A DOC2 protein identified by mutational profiling is essential for apicomplexan parasite exocytosis. Science 335, 218-221 (2012).

40. Triglia, T., Duraisingh, M. T., Good, R. T. \& Cowman, A. F. Reticulocytebinding protein homologue 1 is required for sialic acid-dependent invasion into human erythrocytes by Plasmodium falciparum. Mol. Microbiol. 55, 162-174 (2005).

41. Trager, W. \& Jensen, J. B. Human malaria parasites in continuous culture. Science 193, 673-675 (1976).

42. Lambros, C. \& Vanderberg, J. P. Synchronization of Plasmodium falciparum erythrocytic stages in culture. J. Parasitol. 65, 418-420 (1979).

43. Aley, S. B., Sherwood, J. A., Marsh, K., Eidelman, O. \& Howard, R. J. Identification of isolate-specific proteins on sorbitol-enriched Plasmodium falciparum infected erythrocytes from Gambian patients. Parasitology 92(Pt 3): 511-525 (1986)

44. Kutner, S., Breuer, W. V., Ginsburg, H., Aley, S. B. \& Cabantchik, Z. I. Characterization of permeation pathways in the plasma membrane of human erythrocytes infected with early stages of Plasmodium falciparum: association with parasite development. J. Cell Physiol. 125, 521-527 (1985).

45. Heidrich, H. G. Isolation and functional characterization of Plasmodium falciparum merozoite antigens. Biol. Cell 64, 205-214 (1988).

46. Blackman, M. J. Purification of Plasmodium falciparum merozoites for analysis of the processing of merozoite surface protein-1. Methods Cell Biol. 45, 213-220 (1994).
47. Shimizu, S., Shimura, K., Ito, S. \& Minami, T. Babesia ovata: isolation from erythrocytes and development of an enzyme-linked immunosorbent assay for detection of antibodies. Parasitol. Res. 78, 684-688 (1992).

48. Kuss, C. et al. Quantitative proteomics reveals new insights into erythrocyte invasion by Plasmodium falciparum. Mol. Cell Proteomics 11, 010645 (2012).

49. Wiser, M. F. \& Plitt, B. Plasmodium berghei, P. chabaudi, and P. falciparum: similarities in phosphoproteins and protein kinase activities and their stage specific expression. Exp. Parasitol. 64, 328-335 (1987).

50. Sim, B. K., Toyoshima, T., Haynes, J. D. \& Aikawa, M. Localization of the 175-kilodalton erythrocyte binding antigen in micronemes of Plasmodium falciparum merozoites. Mol. Biochem. Parasitol. 51, 157-159 (1992).

51. Niang, M., Yan Yam, X. \& Preiser, P. R. The Plasmodium falciparum STEVOR multigene family mediates antigenic variation of the infected erythrocyte. PLoS Pathog. 5, e1000307 (2009).

\section{Acknowledgements}

We thank all the blood donors. We thank Dr. Tim Gilberger and his laboratory for technical support of live video microscopy. We thank MR4 for providing us with rabbit anti-EBA175 polyclonal antibodies (contributed by Professor John Adams) and monoclonal antibody g12 (contributed by M. Wiser). We thank Dr. Mike Blackman for PfMSP1 antibodies. T994ARH1 and anti-nucleoporin 100 (Nup 100) antibodies were kind gifts from Dr. Alan Cowman, Australia. We also thank Dr. David Narum for antiEBA175 mAb R215. This research is supported by the Singapore Ministry of Health's National Medical Research Council under its Individual Research Grant (NMRC/1308/ 2011) and Singapore Biomedical Research Council (08/1/22/19/581).

\section{Author contributions}

X.G. and K.G. contributed extensively to the experiments, analysed the data and wrote the manuscript. X.G. and P.R.P. conceived the experiments. S.S.L.Y. performed the epitope mapping experiment and analysed the data. P.R.P. supervised the project, analysed the data and wrote the manuscript.

\section{Additional information}

Supplementary Information accompanies this paper at http://www.nature.com/ naturecommunications

Competing financial interests: The authors declare no competing financial interests.

Reprints and permission information is available online at http://npg.nature.com/ reprintsandpermissions/

How to cite this article: Gao, X. et al. Triggers of key calcium signals during erythrocyte invasion by Plasmodium falciparum. Nat. Commun. 4:2862 doi: 10.1038/ncomms3862 (2013).

(c) $($ This work is licensed under a Creative Commons AttributionNonCommercial-NoDerivs 3.0 Unported License. To view a copy of this license, visit http://creativecommons.org/licenses/by-nc-nd/3.0/ 